\title{
Response profiles of oxygen uptake efficiency during exercise in healthy children
}

Citation for published version (APA):

Bongers, B. C., Hulzebos, E. H. J., Helbing, W. A., ten Harkel, A. D. J., van Brussel, M., \& Takken, T. (2016). Response profiles of oxygen uptake efficiency during exercise in healthy children. European Journal of Preventive Cardiology, 23(8), 865-873. https://doi.org/10.1177/2047487315611769

Document status and date:

Published: 01/05/2016

DOI:

10.1177/2047487315611769

Document Version:

Publisher's PDF, also known as Version of record

Document license:

Taverne

Please check the document version of this publication:

- A submitted manuscript is the version of the article upon submission and before peer-review. There can be important differences between the submitted version and the official published version of record.

People interested in the research are advised to contact the author for the final version of the publication, or visit the DOI to the publisher's website.

- The final author version and the galley proof are versions of the publication after peer review.

- The final published version features the final layout of the paper including the volume, issue and page numbers.

Link to publication

\footnotetext{
General rights rights.

- You may freely distribute the URL identifying the publication in the public portal. please follow below link for the End User Agreement:

www.umlib.nl/taverne-license

Take down policy

If you believe that this document breaches copyright please contact us at:

repository@maastrichtuniversity.nl

providing details and we will investigate your claim.
}

Copyright and moral rights for the publications made accessible in the public portal are retained by the authors and/or other copyright owners and it is a condition of accessing publications that users recognise and abide by the legal requirements associated with these

- Users may download and print one copy of any publication from the public portal for the purpose of private study or research.

- You may not further distribute the material or use it for any profit-making activity or commercial gain

If the publication is distributed under the terms of Article $25 \mathrm{fa}$ of the Dutch Copyright Act, indicated by the "Taverne" license above, 


\section{Response profiles of oxygen uptake efficiency during exercise in healthy children}

\author{
Bart C Bongers ${ }^{1,2}$, Erik HJ Hulzebos², Willem A Helbing ${ }^{3}$, \\ Arend DJ ten Harkel ${ }^{4}$, Marco van Brussel ${ }^{2}$ and Tim Takken ${ }^{2}$
}

European Journal of Preventive Cardiology

2016, Vol. 23(8) 865-873

(C) The European Society of Cardiology 2015

Reprints and permissions: sagepub.co.uk/journalsPermissions.nav DOI: 10.1 I77/20474873 I56II769 ejpc.sagepub.com

(SAGE

\begin{abstract}
Background: Oxygen uptake efficiency (OUE), the relation between oxygen uptake $\left(\mathrm{VO}_{2}\right)$ and minute ventilation $(\mathrm{VE})$, differs between healthy children and children with heart disease. This study aimed to investigate the normal response profiles of OUE during a progressive cardiopulmonary exercise test.

Design: Cross-sectional.

Methods: Healthy children between eight and 19 years of age (I I 4 boys and 100 girls, mean \pm SD age $12.7 \pm 2.8$ years) performed a maximal cardiopulmonary exercise test. Peak $\mathrm{VO}_{2}\left(\mathrm{VO}_{2 \text { peak }}\right)$, ventilatory threshold and peak VE were determined. OUE was determined by the OUE plateau (OUEP), OUE at the ventilatory threshold (OUE@VT) and OUE slope (OUES).

Results: OUEP (42.4 \pm 4.6$)$ and OUE@VT $(41.9 \pm 4.7)$ were similar and less variable than OUES $(2138 \pm 703)$. OUEP correlated strongly with OUE@VT $(r=0.974)$; however, OUEP was weak-to-moderately correlated with $V_{2} O_{2 \text { eak }}$ $(r=0.646)$, the ventilatory threshold $(r=0.548)$ and OUES $(r=0.589)$. OUES correlated strongly with $V_{2}{ }_{2 \text { peak }}$ $(r=0.948)$ and the ventilatory threshold $(r=0.856)$. Reference centiles for OUEP show an almost linear increase from about 37 in eight-year olds to about 47 in 18-year olds, with no sex-difference. OUES increased from about 1400 in eight-year-old boys to approximately 3500 in 18-year-old boys. OUES increased from roughly 1250 in eight-yearold girls to about 2650 in 18-year-old girls.

Conclusions: This study provides sex- and age-related normative values for both OUEP and OUES, which facilitates the interpretation of OUE in children. OUEP and OUES are objective and non-invasive cardiopulmonary exercise test parameters which do not require a maximal effort and might be indicative of cardiorespiratory function during exercise.
\end{abstract}

\title{
Keywords
}

Exercise testing, child, oxygen uptake efficiency plateau, oxygen uptake efficiency slope, cardiovascular health, reference values

Received 27 July 2015; accepted 24 September 2015

\section{Introduction}

Measuring oxygen uptake $\left(V \mathrm{O}_{2}\right)$, carbon dioxide output $\left(V \mathrm{CO}_{2}\right)$, minute ventilation $(V \mathrm{E})$ and heart rate (HR) during an incremental cardiopulmonary exercise test (CPET) up to maximal exertion provides a non-invasive assessment of the integrated physiological response of the pulmonary, cardiovascular and metabolic system to progressive exercise. The non-invasive and dynamic nature of the performed measurements during the progressive load on the cardiorespiratory system provides clinicians with
'Department of Epidemiology, School for Public Health and Primary Care (CAPHRI), Maastricht University, The Netherlands

${ }^{2}$ Child Development \& Exercise Centre, Wilhelmina Children's Hospital, University Medical Centre Utrecht, The Netherlands

${ }^{3}$ Department of Paediatrics, Division of Paediatric Cardiology, Erasmus MC, Sophia Children's Hospital, Rotterdam, The Netherlands ${ }^{4}$ Department of Paediatric Cardiology, Leiden University Medical Centre, The Netherlands

\section{Corresponding author:}

Bart C Bongers, Maastricht University, Department of Epidemiology, UNS40, room 3.554, P.O. Box 616, 6200 MD Maastricht, The Netherlands.

Email: bart.bongers@maastrichtuniversity.nl

Twitter: @BartBongers 
important information that can be used for diagnostic, prognostic and evaluative purposes. Evaluating physiological parameters obtained at specific time points during the CPET (e.g. at peak exercise or at the ventilatory threshold) remains the most commonly used clinical test in paediatric exercise medicine. ${ }^{1}$

Assessing only a single value for the parameters of interest leads to substantial loss of physiological data as provided by evaluating cardiopulmonary response profiles throughout the test. ${ }^{1-4}$ Moreover, considering the trending of certain CPET parameters optimizes the use of the enormous amount of data generated during a routine CPET. Evaluating the response profile of certain CPET parameters has been considered as a crucial component of the interpretative strategy. ${ }^{5-7}$

The oxygen uptake efficiency (OUE) measurement, which is the reciprocal of the ventilatory equivalent for oxygen $\left(\mathrm{VE} / \mathrm{VO}_{2}\right)$, provides an estimation of the efficiency of the $V \mathrm{E}$ with respect to $V \mathrm{O}_{2}$. The OUE slope (OUES) concept is based on the curvilinear relationship between the $V \mathrm{E}$ and $V \mathrm{O}_{2}$ throughout a progressive CPET. Baba et al. ${ }^{8}$ introduced a logarithmic transformation of $V \mathrm{E}$ over the entire exercise period, resulting in a linear relationship between the $V \mathrm{E}$ and $V \mathrm{O}_{2}$ during the last part of the CPET. The regression coefficient of the regression line describing this linear relationship represents the OUES. More recently, Sun et al. ${ }^{9,10}$ proposed plotting the OUE ratio against time during the CPET. The 90-s average of the highest consecutive OUE values is termed OUE plateau (OUEP), which typically occurs around the ventilatory threshold.

OUES and OUEP have been found indicative of functional impairment and prognosis in adult patients with heart failure. ${ }^{10-15}$ Little is known concerning the normalcy of the trending of OUE measurements during progressive exercise in healthy children. The OUEP has never been investigated in children, and although the OUES is known to differ between healthy children and children with heart disease, ${ }^{8,16,17}$ its prognostic value has never been investigated in children with a chronic disease. Before the prognostic value of OUE measurements can be investigated adequately in paediatric patient populations, knowledge concerning its characteristics in healthy children is essential. Therefore, the current study aims to evaluate the OUE response patterns in a large sample of healthy children.

\section{Methods}

\section{Participants}

Two hundred and fourteen healthy Dutch children, ranging in age from eight to 19 years, performed a maximal effort during the CPET. Children and adolescents were recruited from primary and secondary schools, or were family members of hospital staff. All children were free from cardiovascular, pulmonary, neurological, or musculoskeletal disease. Informed consent was obtained from the parents, as well as from children $\geq 12$ years of age. The study protocol was approved by the Medical Ethics Committees of the Erasmus Medical Centre Rotterdam and the University Medical Centre Utrecht, the Netherlands.

\section{CPET}

All participants performed a progressive CPET up to maximal exertion in upright position on an electronically braked cycle ergometer (Jaeger ER900 (Viasys Healthcare GmbH, Höchberg, Germany) at Erasmus Medical Centre Rotterdam and Lode Corival (Lode BV, Groningen, the Netherlands) at the University Medical Centre Utrecht). Participants performed a three-minute warm-up phase (unloaded cycling), where after the work rate was increased by constant increments of 10,15 or $20 \mathrm{~W} / \mathrm{min}$, depending on the participant's body height $(<125 \mathrm{~cm}$, between 125 and $150 \mathrm{~cm}$, and $>150 \mathrm{~cm}$ respectively) according to the Godfrey protocol. ${ }^{18}$ Throughout the CPET, participants had to maintain a pedalling frequency between 60 and 80 revolutions/min. The protocol continued until the participant's pedalling frequency fell definitely below 60 revolutions/min, despite strong verbal encouragement.

During the CPET, participants breathed through a facemask (Hans Rudolph, Kansas City, MO, USA) connected to a calibrated respiratory gas analysis system (Jaeger Oxycon Champion, Viasys Healthcare $\mathrm{GmbH}$, Höchberg, Germany). Expired gas was passed through a flow meter (Triple V volume transducer), an oxygen analyser and a carbon dioxide analyser. The flow meter and gas analysers were connected to a computer, which calculated breath-by-breath $V \mathrm{E}, V \mathrm{O}_{2}$, $V \mathrm{CO}_{2}$ and the respiratory exchange ratio (RER) averaged at 10 -s intervals. HR was measured by continuous twelve-lead electrocardiography. A test was considered to be at or near the maximal level when participants showed clinical signs of intense effort (e.g. unsteady biking, sweating, and clear unwillingness to continue exercising despite strong encouragement), were unable to maintain the required pedalling speed, and when at least one of the following criteria was met: an HR at peak exercise $\left(\mathrm{HR}_{\text {peak }}\right)$ of $>180$ beats $\mathrm{min}^{-1}$ or an RER at peak exercise $\left(\mathrm{RER}_{\text {peak }}\right)$ of $>1.0 .^{19}$

\section{Data analysis}

Absolute values at peak exercise were calculated as the average value over the last $30 \mathrm{~s}$ prior to termination of the test. $\mathrm{HR}_{\text {peak }}$ was defined as the highest $\mathrm{HR}$ achieved during the CPET. The ventilatory threshold was 
defined as the point at which the ventilatory equivalent for oxygen and the partial end-tidal oxygen tension reached a minimum and thereafter began to rise in a consistent manner, coinciding with an unchanged ventilatory equivalent for carbon dioxide and partial endtidal carbon dioxide tension. ${ }^{5}$ When this ventilatory equivalents method appeared to provide uncertain results for a participant's ventilatory threshold, the point at which the linear slope of the relation between the $V \mathrm{CO}_{2}$ and $V \mathrm{O}_{2}$ changed was taken as the ventilatory threshold, according to the V-slope method. ${ }^{20}$ The ventilatory threshold was expressed as an absolute value and as a percentage of $V \mathrm{O}_{2 \text { peak }}$. The graphical presentation of $V \mathrm{E}$ as a function of $V \mathrm{CO}_{2}$ during the progressive CPET was used to determine the point at which $V E$ increased out of proportion to $V \mathrm{CO}_{2}$, the respiratory compensation point. The slope of the relation between $V \mathrm{E}$ and $V \mathrm{CO}_{2}\left(V \mathrm{E} / V \mathrm{CO}_{2}\right.$-slope) was calculated by linear least squares regression of the relation between $V \mathrm{E}$ and $V \mathrm{CO}_{2}$ up to the respiratory compensation point. OUE throughout the CPET was graphically presented by dividing each $V \mathrm{O}_{2}$ value $(\mathrm{ml} / \mathrm{min})$ by the corresponding $V \mathrm{E}$ value $(1 / \mathrm{min})$. The OUEP was then determined as the 90 -s average of the highest consecutive values for OUE $\left(V \mathrm{O}_{2} / V \mathrm{E}\right.$, see Figure 1). ${ }^{9,10}$ OUE at the ventilatory threshold (OUE@VT) was the 60-s average of consecutive values at and immediately before the ventilatory threshold. ${ }^{9}$ Finally, the OUES was calculated using all exercise data by a linear least squares regression of the $V \mathrm{O}_{2}(\mathrm{ml} / \mathrm{min})$ on the common logarithm of the $V \mathrm{E}(1 / \mathrm{min}){ }^{8}$

\section{Statistical analysis}

All data were analysed using the Statistical Package for the Social Sciences for Windows (version 20.0; IBM, SPSS Inc., Chicago, IL, USA). Data are presented as mean value \pm standard deviation (SD) and corresponding ranges. Variability of OUE measurements was evaluated by calculating the coefficient of variation, defined as $100 \times(\mathrm{SD} /$ mean $)$, for the OUES, OUEP and OUE@VT. Independent samples $t$-tests were used to examine differences between boys and girls. Pearson correlation coefficients were calculated to examine associations between OUE measurements and different anthropometric and exercise parameters. Age- and sexrelated reference centiles for the OUES and OUEP were derived using the lambda, mu, sigma (LMS) method as introduced by Cole, ${ }^{21,22}$ using Growth Analyser 3.5 (Growth Analyser BV, Rotterdam, the Netherlands). Based on these reference centiles, prediction equations were established for the OUES and OUEP with sex and age as independent predictors. Significance was set a priori at the 0.05 level.

\section{Results}

Two hundred and fourteen healthy, non-athletic Dutch children, 114 boys and 100 girls, performed a maximal effort during the CPET. Participant characteristics and CPET results are depicted in Table $1 .{ }^{23,24}$ All tests were completed without adverse effects, such as dizziness, fainting or vomiting.

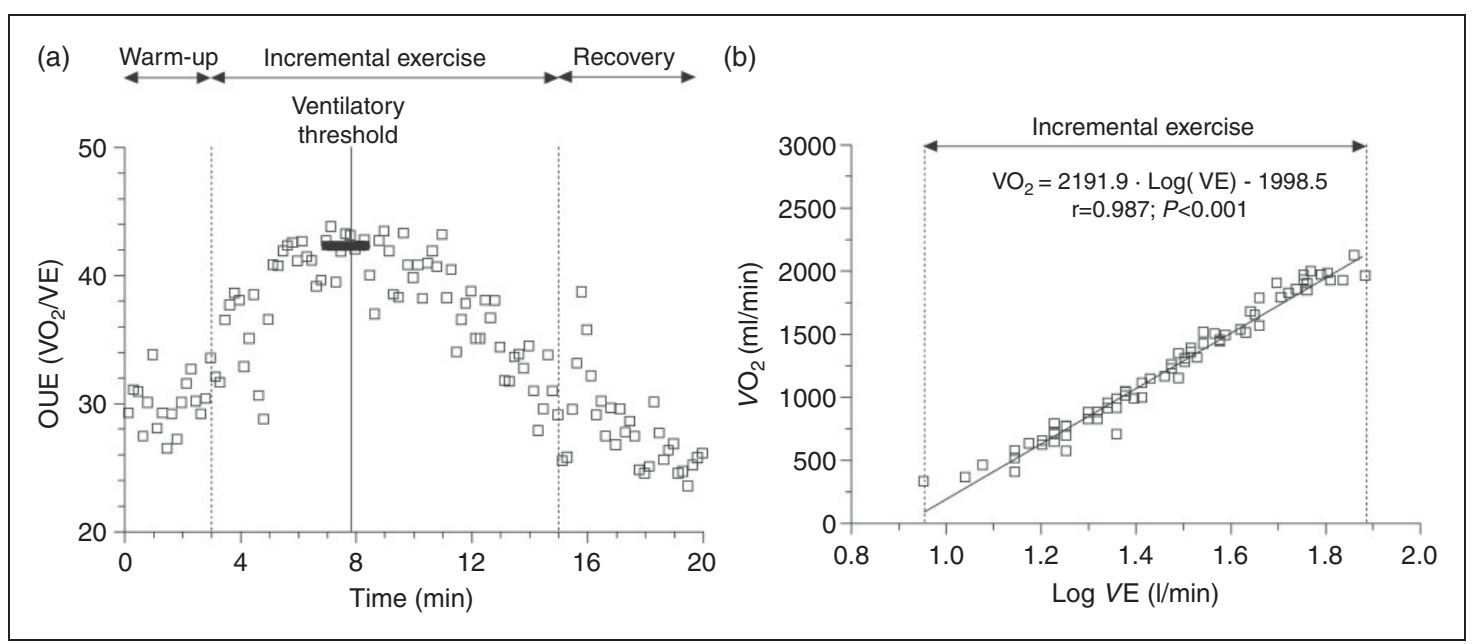

Figure I. OUE measurements throughout a CPET in a I3-year-old healthy girl: the OUEP (graph a) occurred around the ventilatory threshold and was 42.3 (horizontal black bar), whereas the value of the OUES (graph b) was 2191.9.

CPET: cardiopulmonary exercise test; Log VE: common logarithm of the minute ventilation (I/min); OUE: oxygen uptake efficiency; OUEP: oxygen uptake efficiency plateau; OUES: oxygen uptake efficiency slope; VE: minute ventilation $(\mathrm{I} / \mathrm{min})$; VO $\mathrm{O}_{2}$ : oxygen uptake (ml/min) 
Table I. Participant characteristics and CPET results.

\begin{tabular}{|c|c|c|c|c|c|c|}
\hline & Boys $(n=114)$ & & Girls $(n=100)$ & & $p$-value & \\
\hline \multicolumn{7}{|l|}{ Anthropometric parameters: } \\
\hline Age, years & $12.7 \pm 2.9$ & $(8.0-18.5)$ & $12.6 \pm 2.8$ & $(8.0-18.2)$ & $0.84 I$ & \\
\hline Body mass, kg & $47.1 \pm 14.8$ & $(24.1-84.0)$ & $47.6 \pm 13.4$ & $(25.0-81.7)$ & 0.816 & \\
\hline Body mass for age, SDS ${ }^{a}$ & $0.03 \pm 0.83$ & $(-2.13-2.18)$ & $0.19 \pm 0.92^{c}$ & $(-1.96-2.74)$ & $\mathrm{N} / \mathrm{A}$ & \\
\hline Body height, $\mathrm{cm}$ & $158 \pm 17$ & $(124-197)$ & $157 \pm 13$ & $(|28-| 79)$ & 0.550 & \\
\hline Body height for age, SDS ${ }^{a}$ & $0.00 \pm 0.86$ & $(-1.91-2.15)$ & $0.30 \pm 0.89^{d}$ & $(-1.43-2.93)$ & $\mathrm{N} / \mathrm{A}$ & \\
\hline BMI, $\mathrm{kg} / \mathrm{m}^{2}$ & $18.3 \pm 2.5$ & $(13.7-27.2)$ & $18.9 \pm 3.2$ & $(13.7-30.1)$ & 0.113 & \\
\hline $\mathrm{BMI}$ for age, $\mathrm{SDS}^{\mathrm{a}}$ & $0.04 \pm 0.89$ & $(-I .8 I-2.5 I)$ & $0.06 \pm 1.01$ & $(-2.5 \mathrm{I}-2.70)$ & N/A & \\
\hline $\mathrm{BSA}^{\mathrm{b}}, \mathrm{m}^{2}$ & $1.43 \pm 0.30$ & $(0.92-2.07)$ & $1.43 \pm 0.26$ & $(0.95-2.02)$ & 0.927 & \\
\hline \multicolumn{7}{|l|}{ CPET parameters: } \\
\hline$W R_{\text {peak }}, W$ & $183 \pm 68$ & $(75-400)$ & $16 \mid \pm 57$ & $(70-400)$ & 0.011 & $*$ \\
\hline$W R_{\text {peak }}, W / k g$ & $3.8 \pm 0.7$ & $(2.6-5.6)$ & $3.4 \pm 0.6$ & $(2.2-5.3)$ & $<0.001$ & *** \\
\hline $\mathrm{HR}_{\text {peak }}$, beats/min & $189 \pm 9$ & $(|7|-2 \mid I)$ & $190 \pm 9^{g}$ & $(|7|-2 \mid 2)$ & 0.536 & \\
\hline RER $_{\text {peak }}$ & $1.15 \pm 0.07^{\mathrm{e}}$ & $(0.98-1.34)$ & $\mathrm{I} .17 \pm 0.08^{\mathrm{h}}$ & $(1.01-1.37)$ & 0.052 & \\
\hline$V O_{2 \text { peak }}, 1 / \min$ & $2.25 \pm 0.74^{\mathrm{e}}$ & $(1.13-4.10)$ & $\mathrm{I} .95 \pm 0.6 \mathrm{I}^{\mathrm{h}}$ & $(0.97-4.15)$ & 0.001 & $* *$ \\
\hline$V O_{2 \text { peak }}, \mathrm{ml} / \mathrm{kg}$ per min & $48.3 \pm 6.2^{e}$ & $(34.2-62.3)$ & $4 \mathrm{I} .2 \pm 5.7^{\mathrm{h}}$ & $(28.4-55.6)$ & $<0.00$ I & $* * *$ \\
\hline Ventilatory threshold, $1 / \mathrm{min}$ & $1.33 \pm 0.46^{f}$ & $(0.53-2.77)$ & $1.16 \pm 0.37^{h}$ & $(0.56-2.91)$ & 0.003 & $* *$ \\
\hline Ventilatory threshold, $\% V_{2 \text { peak }}$ & $59 \pm 8^{f}$ & $(40-86)$ & $60 \pm 9^{h}$ & $(40-8 I)$ & 0.411 & \\
\hline$V E_{\text {peak }}, 1 / \min$ & $80 \pm 25^{e}$ & $(42-157)$ & $7 I \pm 2 I^{h}$ & $(34-152)$ & 0.007 & $* *$ \\
\hline$V E_{\text {peak }}, \mathrm{l} / \mathrm{kg}$ per min & $1.7 \pm 0.3^{e}$ & $(0.9-2.5)$ & $1.5 \pm 0.3^{h}$ & $(0.8-2.1)$ & $<0.00$ I & $* * *$ \\
\hline VE/VCO ${ }_{2}$-slope & $26.6 \pm 3.4^{\mathrm{e}}$ & $(\mid 4.9-35.1)$ & $27.0 \pm 3.4^{\mathrm{h}}$ & $(17.3-36.0)$ & 0.370 & \\
\hline OUES & $2284 \pm 764^{\mathrm{e}}$ & $(1158-4256)$ & $1970 \pm 585^{h}$ & $(949-3816)$ & 0.001 & ** \\
\hline OUEP & $42.6 \pm 4.7^{\mathrm{e}}$ & $(32.6-60.9)$ & $42.3 \pm 4.6^{h}$ & $(31.3-58.4)$ & 0.686 & \\
\hline OUE@VT & $42.0 \pm 4.6^{\mathrm{e}}$ & $(32.5-58.9)$ & $41.9 \pm 4.7^{\mathrm{h}}$ & $(30.9-57.8)$ & 0.845 & \\
\hline
\end{tabular}

Values are presented as mean \pm SD (range).

${ }^{a}$ Calculated using Dutch normative values. ${ }^{23}$

${ }^{\mathrm{b}}$ Calculated using the equation of Haycock et al. ${ }^{24}$

'Significantly different from $0(p=0.045)$.

dSignificantly different from $0(p=0.00 \mathrm{I})$.

e Respiratory gas analysis measurements were invalid in two boys, so in this case $n=112$.

${ }^{f}$ Respiratory gas analysis measurements were invalid in two boys and the ventilatory threshold was not determinable in one boy, so in this case $n=1 \mathrm{II}$. ${ }^{8}$ Heart rate could not be measured in one girl, so in this case $n=99$.

${ }^{h}$ Respiratory gas analysis measurements were invalid in two girls, so in this case $n=98$.

CPET: cardiopulmonary exercise test; SDS: standard deviation score; N/A: not applicable; BMI: body mass index; BSA: body surface area; WR $\mathrm{R}_{\text {peak: }}$ peak work rate; $\mathrm{HR}_{\text {peak }}$ : heart rate at peak exercise; $\mathrm{RER}_{\text {peak: }}$ respiratory exchange ratio at peak exercise; $\mathrm{VO}_{2 \text { peak: }}$ oxygen uptake at peak exercise; $V \mathrm{E}_{\text {peak: }}$ : minute ventilation at peak exercise; $\mathrm{VE} / \mathrm{VCO}_{2}$-slope: slope of the relation between minute ventilation and carbon dioxide production up to the respiratory compensation point; OUES: oxygen uptake efficiency slope; OUEP: oxygen uptake efficiency plateau; OUE@VT: oxygen uptake efficiency at the ventilatory threshold

$*_{p}<0.05$

$* * p<0.01$

$* * * p<0.001$

OUES, OUEP and OUE@VT values were obtained in all participants. The average OUES, OUEP and OUE@VT values were $2138 \pm 703$ (range: 949 to 4256), $42.4 \pm 4.6$ (range: 31.3 to 60.9 ) and $41.9 \pm 4.7$ (range: 30.9 to 58.9 ), respectively. Coefficients of variation equalled $32.9 \%, 10.9 \%$ and $11.1 \%$ for the OUES, OUEP and OUE@VT, respectively. Even when OUES values were normalized for body mass $(45.9 \pm 7.9)$ or BSA $(1483 \pm 272)$, the variability of the OUES was higher $(17.2 \%$ and $18.3 \%$, respectively) than for the OUEP and the OUE@VT.

Figure 2 presents reference centiles for absolute OUES values in boys (left upper graph) and girls (left lower graph) from eight to 18 years of age. Mean absolute OUES values increase from approximately 1400 at eight years of age to 3500 at 18 years of age in boys $(+150 \%$, Figure 2; Supplementary Table 1 in Supplementary Material online), whereas values 

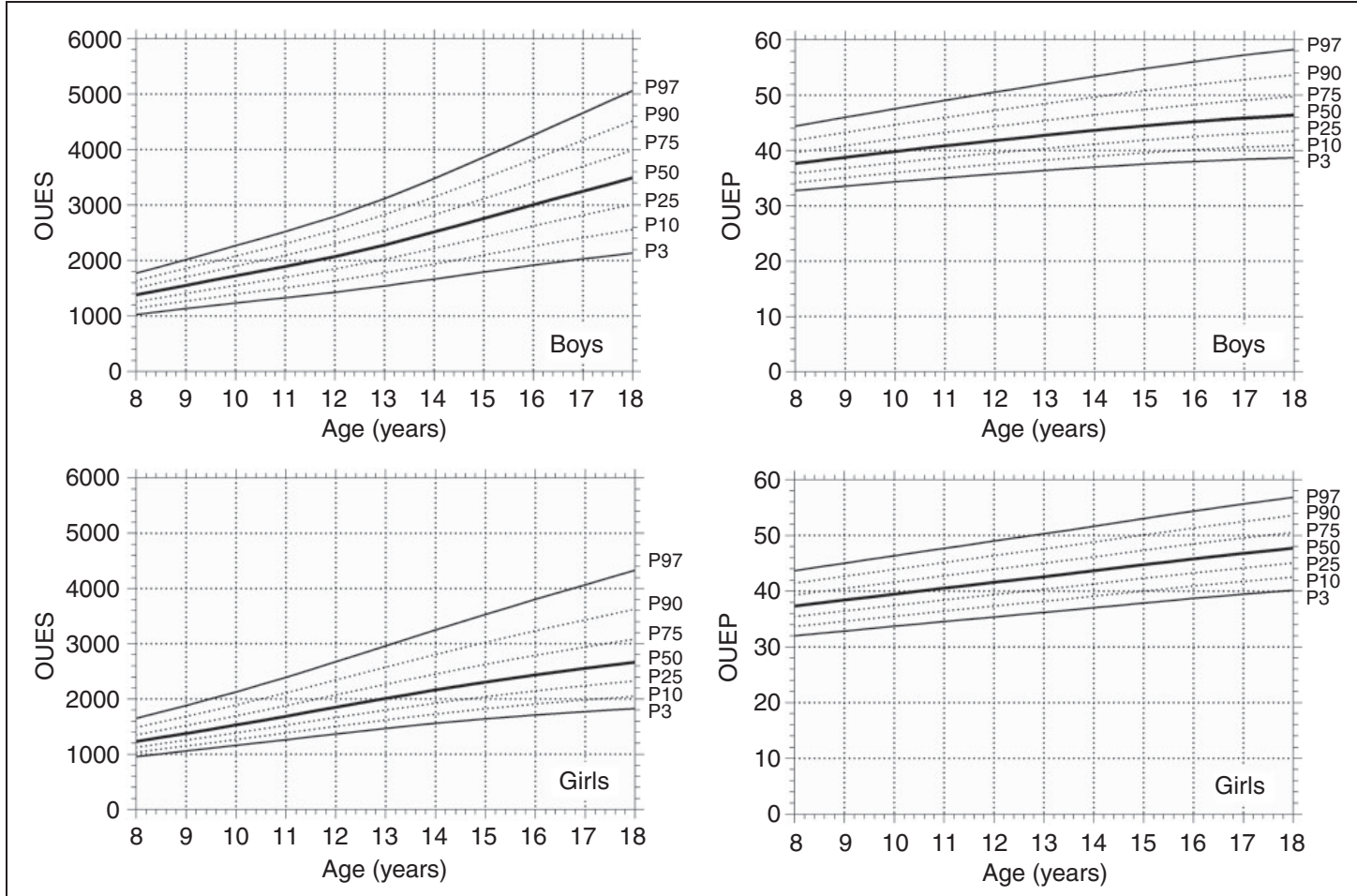

Figure 2. Age- and sex-related reference centiles for OUE: the OUES against age in boys (left upper graph) and girls (left lower graph) and the OUEP against age in boys (right upper graph) and girls (right lower graph).

OUE: oxygen uptake efficiency; OUEP: oxygen uptake efficiency plateau; OUES: oxygen uptake efficiency slope

Table 2. OUES and OUEP prediction equations for healthy children.

\begin{tabular}{|c|c|c|c|c|c|c|c|}
\hline \multirow{2}{*}{ Dependent } & \multirow[b]{2}{*}{ Sex } & \multicolumn{5}{|c|}{ Constant and independent predictors } & \multirow{2}{*}{$\begin{array}{l}\begin{array}{l}\text { Statistica } \\
\text { analysis }\end{array} \\
R^{2}\end{array}$} \\
\hline & & Constant & Age (years) & & & & \\
\hline \multirow[t]{2}{*}{ OUES $^{\mathrm{a}}$} & Boys & 577.208 & & & $6.172 \times$ age $^{2}$ & $52.069 \times$ age & 0.9997 \\
\hline & Girls & -342.403 & & & $-2.589 \times \operatorname{age}^{2}$ & $214.606 \times$ age & 0.9993 \\
\hline \multirow[t]{2}{*}{ OUES/kg } & Boys & 21.757 & $-0.0011 \times \mathrm{age}^{4}$ & $0.0562 \times \mathrm{age}^{3}$ & $-1.0675 \times \mathrm{age}^{2}$ & $8.8991 \times$ age & 0.9063 \\
\hline & Girls & 41.276 & $-0.0006 \times \mathrm{age}^{4}$ & $0.0247 \times \mathrm{age}^{3}$ & $-0.3252 \times$ age $^{2}$ & I. $4446 \times$ age & 0.9910 \\
\hline \multirow[t]{2}{*}{ OUEP ${ }^{a}$} & Boys & 26.340 & & & $-0.029 \times \mathrm{age}^{2}$ & $1.641 \times$ age & 0.9998 \\
\hline & Girls & 28.437 & & & $-0.0036 \times$ age $^{2}$ & I.1409 $\times$ age & 0.9999 \\
\hline
\end{tabular}

${ }^{\mathrm{a}}$ For the absolute OUES and OUEP, the presented constants and independent predictors are based on polynomial regression (order 2) of the P50 values depicted in Figure 2.

${ }^{b}$ For the OUES $/ \mathrm{kg}$, the presented constants and independent predictors are based on polynomial regression (order 4).

OUE: oxygen uptake efficiency; OUES: oxygen uptake efficiency slope; OUEP: oxygen uptake efficiency plateau.

increase from about 1250 at eight years of age to 2650 at 18 years of age in girls $(+112 \%$, Figure 2; Supplementary Table 1 in Supplementary Material online). Reference centiles for absolute OUEP values in boys (right upper graph) and girls (right lower graph) from eight to 18 years of age are also depicted in Figure 2. Mean OUEP values in both boys and girls increase from roughly 37 at eight years of age up to approximately 47 at 18 years of age $(+27 \%$, Figure 2 ; Supplementary Table 1 in Supplementary Material online), with no significant sex-differences. Table 2 depicts prediction equations for the OUES and the OUEP for healthy children and adolescents.

Correlations (Supplementary Table 2 in Supplementary Material online) between the OUEP and absolute $V \mathrm{O}_{2 \text { peak }}$ and between the OUEP and the 
ventilatory threshold were much lower than those between the OUES and absolute $V \mathrm{O}_{2 \text { peak }}(r=0.646$ vs. $r=0.948$; with $p<0.001$ for both coefficients) and between the OUES and the ventilatory threshold $(r=0.548$ vs. $r=0.856$; with $\mathrm{p}<0.001$ for both coefficients). The $V \mathrm{E} / V \mathrm{CO}_{2}$-slope was moderately to strongly correlated with both the OUEP $(r=-0.719$; $p<0.001)$ and the OUES $(r=-0.641 ; p<0.001)$. The OUEP correlated highly with OUE@VT $(r=0.974$; $p<0.001$ ), whereas the associations between the OUEP and the OUES and between the OUE@VT and the OUES were lower $(r=0.589$ and $r=0.578$, respectively; $p<0.001$ for both coefficients).

\section{Discussion}

This study describes the characteristics of the OUE in a large healthy paediatric population, aged 8-19 years. The OUES and OUEP are easy, non-invasive and objectively determinable from CPET data and provide information about the function of the cardiorespiratory system during progressive exercise. Calculation of the OUES does not require a maximal effort of the child, as the relationship between the common logarithm of the $V \mathrm{E}$ and the $V \mathrm{O}_{2}$ is linear during the last part of the CPET. The OUEP also does not require a maximal effort of the child, as it occurs around the ventilatory threshold at moderate exercise intensity. Similar values were found for the OUEP and OUE@VT, which were less variable between participants than the OUES. These coefficients of variation were slightly lower than those described for healthy adults. ${ }^{9}$ Absolute OUES values increased with age in boys and girls, with higher values attained by boys. This finding is in agreement with previous studies in children. ${ }^{25-27}$ OUEP values also increased with age in boys and girls, with no significant sex-difference. The OUES correlated strongly with $V \mathrm{O}_{2 \text { peak }}$ and the ventilatory threshold in this study, which is in line with previous research in children. 8 ,16,25-29 The OUEP was weak-to-moderately correlated with $V \mathrm{O}_{2 \text { peak }}$, the ventilatory threshold and the OUES, which was also reported in healthy adults by Sun et al. ${ }^{9}$

Sun et al. ${ }^{9}$ reported higher test-retest reproducibility for the OUEP compared with the OUES. The authors reported an average variability between paired values of the OUEP and OUES in 24 healthy adults of $3.9 \pm 2.5 \%$ and $11.3 \pm 8.6 \%$, respectively $(p<0.001)$. Despite this higher variability within participants, the OUES has previously been reported to show good reproducibility in healthy adults. ${ }^{30,31}$ Baba et al. ${ }^{30}$ found a coefficient of repeatability (COR) for the OUES, $V \mathrm{O}_{2 \text { peak }}$ and ventilatory threshold of $20 \%$, $16 \%$ and $31 \%$, respectively. Similarly, van Laethem et al. $^{31}$ reported an intraclass correlation coefficient
(ICC) for the OUES of 0.93 and a COR of $18.7 \%$, which was comparable to $V \mathrm{O}_{2 \text { peak }}$ (ICC of 0.95 and COR of $17.3 \%$ ) and superior to the ventilatory threshold (ICC of 0.86, COR not reported). De Groot et al. ${ }^{32}$ found an ICC of 0.80 and a coefficient of variation within participants of $24.3 \%$ for the OUES in 23 children with spina bifida. Data in children addressing test-retest reproducibility for the OUEP are currently lacking.

The OUES and OUEP are indicative of cardiorespiratory function during exercise. Without significant lung disease, both the OUES and OUEP are indicative of cardiovascular function throughout exercise. The curvilinear response of $V \mathrm{E}$ during progressive exercise, caused by the progressively increasing contribution of the anaerobic glycolysis to energy metabolism, provides the conceptual basis for the OUES. Baba et al. ${ }^{8}$ observed that the logarithmic transformation of the $V \mathrm{E}$ makes the relation between $V \mathrm{E}$ and $V \mathrm{O}_{2}$ during progressive exercise linear, in which the slope of the regression line describing this linear relation represents the OUES. In essence, the OUES provides an estimation of the efficiency of the $V \mathrm{E}$ with respect to the $V \mathrm{O}_{2}$. Higher OUES values indicate a more efficient $V \mathrm{O}_{2}$. OUEP represents the maximal (most efficient) OUE, which occurs during submaximal exercise intensities around the ventilatory threshold, and not at rest or at maximal exercise. At rest, the high mixed venous oxygen content requires less oxygen extraction from alveolar gas. Moreover, ventilation is less efficient at rest for $V \mathrm{O}_{2}$, as low $V \mathrm{E}$ values result in a high physiologic dead space resulting in suboptimal ventilation perfusion matching. During maximal exercise, mixed venous oxygen content is lowest. Moreover, high $V \mathrm{E}$ values result in an optimal ratio of physiologic dead space to tidal volume and therefore in an optimal ventilation-perfusion matching. However, anaerobic glycolysis, with lactic acid and carbon dioxide as its by-products, results in an excessive ventilatory response to metabolic acidosis. The latter will significantly reduce the ratio of $V \mathrm{O}_{2}$ to $V \mathrm{E}$ (OUE). Therefore, the OUEP typically occurs around the ventilatory threshold, before OUE values decline caused by the increased $V \mathrm{E}$ as a result of anaerobic glycolysis. Higher OUEP values indicate a more efficient $V \mathrm{O}_{2}$, whereas lower values represent a lower $V \mathrm{O}_{2}$ for any given $V E$. Hence, both the OUES and OUEP are dependent on the ventilatory threshold and the ratio of physiologic dead space to tidal volume. ${ }^{8,9}$

By definition, a maximal CPET is highly dependent on the motivation of the child to continue exercising against high intensities, when dyspnoea, muscle fatigue and other stress sensations are commonly experienced. When the delivered effort of the child during a CPET cannot be classified as maximal, the complete test may be deemed a failure despite the wealth of data 
successfully collected. ${ }^{1}$ The latter can provide important information concerning the normalcy of the child's pulmonary, cardiovascular and metabolic response to progressive exercise, even in the absence of a 'true' $V \mathrm{O}_{2 \text { peak }}$. A maximal effort of the child is not required to determine the OUES and the OUEP. This makes OUE measurements an appealing alternative in patients with heart failure or cardiac rhythm abnormalities in which maximal exercise testing is sometimes contraindicated. When the OUES is calculated, the $V E$ is logarithmically transformed to produce a linear slope when plotted against $V \mathrm{O}_{2}$. This makes the OUES theoretically an exercise intensity independent measure which is resistant to disruption by early termination of the CPET. Several studies in children confirmed the linearity of the OUES; ${ }^{16,25-27}$ however, other studies found OUES values calculated using exercise data up to submaximal exercise intensities to be slightly, but significantly, lower than OUES values calculated using exercise data up to peak exercise. ${ }^{8,28,33}$ The OUEP occurs around the ventilatory threshold, just before OUE values start to decline due to the increased $V \mathrm{E}$ as a result of the bicarbonate buffering of the proton from lactate resulting in the nonmetabolic production of carbon dioxide. Hence, OUE measurements can be calculated from submaximal exercise testing as long as children are able to reach their ventilatory threshold. If the child does not reach his or her ventilatory threshold, OUEP values should be interpreted with caution. OUES values should be interpreted with caution when the ventilatory threshold is not reached or when a plateau in $V \mathrm{O}_{2}$ is observed despite an increase in work rate $\left(V \mathrm{O}_{2 \max }\right) \cdot{ }^{34}$

Despite the fact that the ventilatory threshold involves only a single value of a CPET, it has been found to be strongly correlated with $V \mathrm{O}_{2 \text { peak. }} \cdot{ }^{35}$ This makes the ventilatory threshold a useful indicator for aerobic exercise capacity in children unable or unwilling to perform a maximal effort. An important shortcoming of the ventilatory threshold is the fact that it is sometimes not clearly identifiable, as well as the continuing debate concerning its reproducibility. ${ }^{36}$ The ventilatory threshold depends on the mode of exercise testing, the utilized exercise protocol and the method of detection, and is a subjective measurement and is thus subject to substantial intra- and inter-observer variability. ${ }^{37-40}$ Both the OUES and OUEP are easy determinable in each CPET and free from interobserver and intraobserver variability, since they are mathematically determined by a set of CPET data.

\section{Study limitations}

Reference centiles were generated with respect to age and sex, without taking other relevant factors (e.g. race, maturation and anthropometrics) into account.
Moreover, we did not measure physical activity levels of the included participants. Finally, a longitudinal study design would have provided a more secure analysis of the development of OUE throughout the paediatric age range.

\section{Future research}

It is currently unknown whether the OUEP is able to differentiate between healthy children and children with cardiovascular or severe respiratory disease (forced expiratory volume in $1 \mathrm{~s}<70 \%$ of predicted). Previous findings suggest that the OUEP has discriminative and prognostic value in adults with heart disease. ${ }^{10}$ Further research is required to assess its discriminative and prognostic properties in different paediatric patient populations. Test-retest reproducibility, as well as the evaluative properties of the OUES and OUEP, in paediatric populations remains also the subject of further research.

\section{Conclusion}

This study provides sex- and age-related normative values for both OUEP and OUES, which facilitates the interpretation of OUE in children. The OUEP and OUES increase with age in boys and girls, in which boys attain higher OUES values than girls. Both the OUEP and OUES are objective and non-invasive CPET parameters which do not require a maximal effort and might be indicative of cardiorespiratory function during exercise. From OUE measurements, the OUES seems to be a valid submaximal alternative for $V \mathrm{O}_{2 \text { peak }}$.

\section{Declaration of conflicting interests}

The author(s) declared no potential conflicts of interest with respect to the research, authorship, and/or publication of this article.

\section{Funding}

The author(s) received no financial support for the research, authorship, and/or publication of this article.

\section{References}

1. Cooper DM, Leu SY, Galassetti P, et al. Dynamic interactions of gas exchange, body mass, and progressive exercise in children. Med Sci Sports Exerc 2014; 46: 877-886.

2. Neder JA, Nery LE, Peres C, et al. Reference values for dynamic responses to incremental cycle ergometry in males and females aged 20 to 80. Am J Respir Crit Care Med 2001; 164: 1481-1486.

3. Sun XG, Hansen JE, Garatachea N, et al. Ventilatory efficiency during exercise in healthy subjects. Am J Respir Crit Care Med 2002; 166: 1443-1448. 
4. Ramos RP, Alencar MC, Treptow E, et al. Clinical usefulness of response profiles to rapidly incremental cardiopulmonary exercise testing. Pulm Med 2013; 359021.

5. American Thoracic Society; American College of Chest Physicians. ATS/ACCP statement on cardiopulmonary exercise testing. Am J Respir Crit Care Med 2003; 167: 211-277.

6. Palange $\mathrm{P}$, Ward SA, Carlsen $\mathrm{KH}$, et al. Recommendations on the use of exercise testing in clinical practice. Eur Respir J 2007; 29: 185-209.

7. Wasserman K, Hansen JE, Sue DY, et al. Principles of exercise testing and interpretation: Including pathophysiology and clinical applications. 5th ed. Philadelphia: Lippincott Williams \& Wilkins, 2012.

8. Baba R, Nagashima M, Goto M, et al. Oxygen uptake efficiency slope: A new index of cardiorespiratory functional reserve derived from the relation between oxygen uptake and minute ventilation during incremental exercise. J Am Coll Cardiol 1996; 28: 1567-1572.

9. Sun XG, Hansen JE and Stringer WW. Oxygen uptake efficiency plateau: Physiology and reference values. Eur J Appl Physiol 2012; 112: 919-928.

10. Sun XG, Hansen JE and Stringer WW. Oxygen uptake efficiency plateau best predicts early death in heart failure. Chest 2012; 141: 1284-1294.

11. Davies LC, Wensel R, Georgiadou P, et al. Enhanced prognostic value from cardiopulmonary exercise testing in chronic heart failure by non-linear analysis: Oxygen uptake efficiency slope. Eur Heart J 2006; 27: 684-690.

12. Arena R, Brubaker P, Moore B, et al. The oxygen uptake efficiency slope is reduced in older patients with heart failure and a normal ejection fraction. Int $J$ Cardiol 2010; 144: 101-102.

13. Arena R, Myers J, Abella J, et al. Prognostic significance of the oxygen uptake efficiency slope: Percent-predicted versus actual value. Am J Cardiol 2010; 105: 757-758.

14. Antoine-Jonville S, Pichon A, Vazir A, et al. Oxygen uptake efficiency slope, aerobic fitness, and V(E)-VCO2 slope in heart failure. Med Sci Sports Exerc 2012; 44: 428-434.

15. Arena R, Guazzi M, Myers J, et al. The relationship between minute ventilation and oxygen consumption in heart failure: Comparing peak $\mathrm{VE} / \mathrm{VO}_{2}$ and the oxygen uptake efficiency slope. Int J Cardiol 2012; 154: 384-385.

16. Bongers BC, Hulzebos HJ, Blank AC, et al. The oxygen uptake efficiency slope in children with congenital heart disease: Construct and group validity. Eur J Cardiovasc Prev Rehabil 2011; 18: 384-392.

17. Motonaga KS, Punn R, Axelrod DM, et al. Diminished exercise capacity and chronotropic incompetence in pediatric patients with congenital complete heart block and chronic right ventricular pacing. Heart Rhythm 2015; 12: $560-565$.

18. Godfrey S. Exercise testing in children: Applications in health and disease. London: WB Saunders Company Ltd, 1974.

19. Armstrong $\mathrm{N}$ and Welsman JR. Aerobic fitness. In: Armstrong $\mathrm{N}$ and van Mechelen W (eds) Paediatric exercise science and medicine. Oxford: Oxford University Press, 2008, pp.97-108.
20. Beaver WL, Wasserman K and Whipp BJ. A new method for detecting anaerobic threshold by gas exchange. $J$ Appl Physiol 1986; 60: 2020-2027.

21. Cole TJ. Fitting smoothed centile curves to reference data. J R Stat Soc 1988; 151: 385-418.

22. Cole TJ. The LMS method for constructing normalized growth standards. Eur J Clin Nutr 1990; 44: 45-60.

23. Fredriks AM, van Buuren S, Wit JM, et al. Body index measurements in 1996-7 compared with 1980. Arch Dis Child 2000; 82: 107-112.

24. Haycock GB, Schwartz GJ and Wisotsky DH. Geometric method for measuring body surface area: A height-weight formula validated in infants, children, and adults. J Pediatr 1978; 93: 62-66.

25. Marinov B and Kostianev S. Exercise performance and oxygen uptake efficiency slope in obese children performing standardized exercise. Acta Physiol Pharmacol Bulg 2003; 27: 59-64.

26. Marinov B, Mandadzhieva S and Kostianev S. Oxygenuptake efficiency slope in healthy 7- to 18-year-old children. Pediatr Exerc Sci 2007; 19: 159-170.

27. Akkerman M, van Brussel M, Bongers BC, et al. Oxygen uptake efficiency slope in healthy children. Pediatr Exerc Sci 2010; 22: 431-441.

28. Bongers BC, Hulzebos EH, Arets BG, et al. Validity of the oxygen uptake efficiency slope in children with cystic fibrosis and mild-to-moderate airflow obstruction. Pediatr Exerc Sci 2012; 24: 129-141.

29. Breithaupt PG, Colley RC and Adamo KB. Using the oxygen uptake efficiency slope as an indicator of cardiorespiratory fitness in the obese pediatric population. Pediatr Exerc Sci 2012; 24: 357-368.

30. Baba R, Kubo N, Morotome Y, et al. Reproducibility of the oxygen uptake efficiency slope in normal healthy subjects. J Sports Med Phys Fitness 1999; 39: 202-206.

31. van Laethem C, de Sutter J, Peersman W, et al. Intratest reliability and test-retest reproducibility of the oxygen uptake efficiency slope in healthy participants. Eur J Cardiovasc Prev Rehabil 2009; 16: 493-498.

32. de Groot JF, Takken T, Gooskens RH, et al. Reproducibility of maximal and submaximal exercise testing in 'normal ambulatory' and 'community ambulatory' children and adolescents with spina bifida: Which is best for the evaluation and application of exercise training? Phys Ther 2011; 91: 267-276.

33. Drinkard B, Roberts MD, Ranzenhofer LM, et al. Oxygen-uptake efficiency slope as a determinant of fitness in overweight adolescents. Med Sci Sports Exerc 2007; 39: 1811-1816.

34. Niemeijer VM, van't Veer M, Schep G, et al. Causes of nonlinearity of the oxygen uptake efficiency slope: A prospective study in patients with chronic heart failure. Eur J Prev Cardiol 2014; 21: 347-353.

35. Hebestreit H, Staschen B and Hebestreit A. Ventilatory threshold: A useful method to determine aerobic fitness in children? Med Sci Sports Exerc 2000; 32: 1964-1969.

36. Bongers BC, van Brussel M, Hulzebos HJ, et al. Pediatric norms for cardiopulmonary exercise testing. In relation to sex and age, 2nd ed. 's Hertogenbosch: Uitgeverij BOXpress, 2014. 
37. Yeh MP, Gardner RM, Adams TD, et al. 'Anaerobic threshold': Problems of determination and validation. J Appl Physiol 1983; 55: 1178-1186.

38. Gladden LB, Yates JW, Stremel RW, et al. Gas exchange and lactate anaerobic thresholds: Inter- and intraevaluator agreement. J Appl Physiol 1985; 58: 2082-2089.
39. Shimizu M, Myers J, Buchanan N, et al. The ventilatory threshold: method, protocol, and evaluator agreement. Am Heart $J$ 1991; 122: 509-516.

40. Vanhees L, Lefevre J, Philippaerts R, et al. How to assess physical activity? How to assess physical fitness? Eur J Cardiovasc Prev Rehabil 2005; 12: 102-114. 